\title{
COMPARAÇÃO DE TAXAS DE EROSÃO EM ESTRADAS FLORESTAIS ESTIMADAS PELO MODELO WEPP (WATER EROSION PREDICTION PROJECT) MODIFICADO EM RELAÇÃO A MEDIÇÕES EXPERIMENTAIS ${ }^{1}$
}

\author{
Carlos Cardoso Machado 2 , Alessandra Reis Garcia ${ }^{3}$, Elias Silva ${ }^{4}$ e Alessandro Machado Fontes ${ }^{5}$
}

\begin{abstract}
RESUMO - O objetivo do trabalho foi testar o modelo WEPP (Water Erosion Prediction Project), através de comparações entre volume de enxurrada e perda de solo observados experimentalmente, provenientes dos segmentos de estradas florestais submetidas à chuva natural com inclinações de 1 e $7 \%$ e comprimentos de rampa de 20 e $40 \mathrm{~m}$, e aqueles preditos pelo aplicativo, visando o desenvolvimento de um modelo brasileiro de predição de erosão em estradas florestais. Na determinação da quantidade do material erodido foram instalados tambores coletores, com capacidade de 209,25 litros, localizados na parte inferior das estradas, onde foram inseridas tubulações de PVC de 2 polegadas para coleta dos sedimentos provenientes da estrada propriamente dita. Nos tambores coletores foram feitos orifícios nivelados e perfeitamente iguais, posicionados a $0,65 \mathrm{~m}$ do fundo do primeiro e a $0,60 \mathrm{~m}$ do fundo do segundo, que funcionaram como um divisor Geib. Nas parcelas de 20 e $40 \mathrm{~m}$ de comprimento foram feitos cinco e sete orifícios, respectivamente, no primeiro e segundo tambores. O terceiro tambor foi utilizado para coletar o excedente da enxurrada proveniente do segundo tambor. Os tambores foram ligados em série, através de cano PVC de 2 polegadas. Os dados de volume e intensidade de precipitação diária foram obtidos com a instalação de pluviômetro e pluviógrafo no local. O período de coleta de dados foi de um ano, concentrando-se na época das chuvas. Posteriormente, os arquivos de clima, precipitação, solo, inclinação e comprimento do segmento foram introduzidos e adaptados ao modelo de predição de erosão WEPP com o propósito de testá-lo, visando a confecção de um modelo apropriado às condições brasileiras.
\end{abstract}

Palavras-chaves: $\quad$ WEPP, estrada florestal, volume de enxurrada e perda de solo.

\section{COMPARISON OF EROSION RATES IN FOREST ROADS APPLYING THE WEPP MODEL (WATER EROSION PREDICTION PROJECT) MODIFIED WITH REFERENCE TO EXPERIMENTAL MEASUREMENTS}

\begin{abstract}
The objective of this work was to test the WEPP model (Water Erosion Prediction Project), by comparing experimentally observed runoff volume and soil loss in forest road segments under normal rainfall, with 1 and $7 \%$ slopes and lengths of 20 and $40 \mathrm{~m}$, and those predicted by the WEPP, aiming to develop a Brazilian model of erosion prediction on forest roads. To determine the amount of eroded matter, 209.25 l capacity collector drums were placed on the lower level of the road segment. PVC pipes of 2 inches were connected to the drums to collect sediments from the road. Openings at the same height and size were made on the drums, located at $0.65 \mathrm{~m}$ from the bottom of the first drum and at $0.60 \mathrm{~m}$ from the bottom of the second drum, which functioned as a Geib divisor. In the 20 and $40 \mathrm{~m}$ long plots five and seven openings were made, respectively, on the first and second drums, with the third drum being used to collect the runoff excess from the second drum. The drums were connected in series through 2 inch - PVC pipes. Data of volume and intensity of daily precipitation were obtained by means of pluviometers and pluviographs. The period of data collection was one year, concentrated in the rainy season. Files on climate, precipitation, soil, slope and segment length were introduced and adapted to the WEPP model erosion prediction model to test it, aiming to elaborate a model suited to the Brazilian forest conditions.
\end{abstract}

Key words: $\quad$ WEPP, forest road, runoff volume and soil loss.

1 Recebido para publicação em 21.1.2002.

Aceito para publicação em 12.5.2033.

Pesquisa financiada pelo CNPq/CAPES/DURATEX.

2 Professor Titular do Departamento de Engenharia Florestal da Universidade Federal de Viçosa - UFV e Pesquisador nível I do CNPq, 36571-000 Viçosa-MG; ${ }^{3}$ Pesquisadora do IBAMA; ${ }^{4}$ Professor Adjunto do Departamento de Engenharia Florestal da $\mathrm{UFV} ;{ }^{5}$ Bolsista de Iniciação Científica da UFV. 


\section{INTRODUÇÃO}

As florestas são um dos maiores recursos naturais em muitas nações. A cobertura florestal está por volta de $30 \%$ da área de terra do Planeta. Elas estão freqüentemente localizadas nas áreas marginais, mas com adequada pluviosidade para o crescimento florestal. Normalmente estas áreas são íngremes e estariam propensas à erosão e instabilidade, se não fosse a cobertura florestal. Geralmente as áreas florestadas têm erosão mínima, exceto se perturbadas. As perturbações incluem o fogo, a colheita e a construção de estradas florestais.

No setor florestal, a rede viária é a principal base de toda a atividade florestal, sobretudo no transporte de matéria-prima; no entanto as estradas florestais têm sido uma das principais causas da erosão e do assoreamento dos cursos d'água nas florestas plantadas.

É imprescindível a realização de estudos relacionados aos aspectos técnicos, econômicos e ambientais, visando alternativas a serem incorporadas como medidas mitigadoras de impactos ambientais.

Nesse contexto, vários pesquisadores, em todas as partes do mundo, têm direcionado seus estudos para a análise e quantificação dos sedimentos advindos da erosão, e os resultados são de fato preocupantes (Fernandes, 1997).

Os estudos qualitativos sobre erosão são válidos para um zoneamento inicial de áreas suscetíveis a esse fenômeno. Entretanto, a não-quantificação das perdas de solo impossibilita uma análise mais aprofundada sobre o grau de degradação por erosão e o seu controle. Além disto, análises puramente qualitativas não permitem comparações de diferentes cenários de planejamento conservacionista, nem fornecem dados para estudos de sedimentação. Desta forma, evidencia-se a necessidade de utilizar ferramentas que levem à quantificação da erosão, o que pode ser obtido através de alguns modelos preditivos (Chaves, 1996).

Os modelos de simulação, como os modelos matemáticos de predição de erosão, são ferramentas poderosas na pesquisa e nas práticas agrícolas, pois quando aplicados a situações de campo auxiliam na determinação das práticas conservacionistas e de manejos mais indicados para os diferentes cenários de aplicação (Chaves, 1996). Se, entretanto, essas determinações usassem apenas resultados experimentais, elas seriam seriamente limitadas tanto em termos de custos e de tempo, como também pela imprevisibilidade da natureza (Ferreira \& Smith, 1988, citados por Fernandes, 1997).

O modelo WEPP, por sua vez, representa a última geração de modelos com base em processos e foi o resultado de um esforço multidisciplinar, envolvendo pesquisadores de várias agências e universidades americanas (Chaves, 1996).

O modelo WEPP se baseia nos fundamentos das teorias de infiltração, física do solo, fitotecnia, hidráulica e mecânica da erosão. Proporciona várias vantagens sobre outras tecnologias de previsão de erosão, como a capacidade de estimar as distribuições espacial e temporal da perda de solo e deposição e da perda de solo líquida na vertente ou em cada ponto dela, tanto em nível diário, quanto mensal ou anual. Além da opção de simulação contínua, o modelo pode também ser usado na opção evento a evento (Chaves, 1992).

A modelagem da erosão está, na maior parte dos processos, orientada e baseada no modelo WEPP. Esse modelo não somente prediz taxas de erosão do solo, mas também a liberação de sedimentos. O Forest Service dos Estados Unidos tem validado com sucesso o modelo WEPP para um grande número de condições de estradas florestais (Elliot et al., 1997).

O projeto WEPP é constituído de três versões básicas: uma versão para vertentes (hillslope version), uma versão para bacias hidrográficas (watershed version) e uma de quadrícula (grid version). Por se tratar de um modelo semideterminístico, o modelo WEPP também pode ser aplicado às condições brasileiras, desde que os arquivos de entrada de dados sofram adaptações e modificações para que reflitam as nossas condições (Angulo Filho et al., 1992).

\subsection{Componentes do modelo WEPP}

O modelo WEPP pode ser dividido conceitualmente em seis componentes: geração de clima, hidrologia, crescimento de plantas, solos, erosão/deposição e irrigação (opcional). Esses componentes serão descritos a seguir, de acordo com Chaves (1992).

\subsubsection{Geração de Clima}

A geração de clima pode ser feita estocasticamente por um submodelo paralelo (CLIGEN), no qual informações sobre volume, duração, intensidade e frequiência de precipitação, temperaturas máxima e mínima, 
radiação solar e ponto de orvalho para a localidade de interesse são geradas com base em séries históricas da estação meteorológica mais próxima. As informações geradas pelo submodelo CLIGEN são gravadas em um arquivo de clima, para posterior utilização pelo modelo WEPP. Dados reais de clima, ou mesmo dados sintéticos, podem também ser diretamente utilizados pelo modelo, sem a necessidade de utilização do CLIGEN.

\subsubsection{Hidrologia}

O componente hidrológico do modelo calcula a infiltração, o escoamento superficial e o balanço hídrico diário, incluindo a evapotranspiração e a percolação profunda. A infiltração é calculada através do método de Green-Ampt, modificado para intensidades variáveis de chuva e tempo de empoçamento. O escoamento superficial é estimado através da equação da onda cinemática para escoamento unidirecional, com solução analítica obtida pelo método das características.

\subsubsection{Crescimento de Plantas}

O componente de crescimento de plantas estima o crescimento, a senescência das plantas e a decomposição dos restos culturais. No caso de culturas anuais, o crescimento das plantas é estimado em função do número de graus-dia e da umidade do solo.

\subsubsection{Solos}

Muitos parâmetros de solo usados nos componentes hidrológicos e de erosão são dinâmicos em função das operações de cultivo, intemperismo e histórico da precipitação, e são reajustados, automaticamente, em função do tempo, das operações de cultivo e dos processos hidrológicos.

\subsubsection{Erosão}

O componente de erosão do modelo WEPP utiliza a equação permanente de continuidade de sedimento como base dos cálculos de erosão. A erosão do solo é dividida em erosão entre sulcos e erosão em sulcos. A primeira é causada pelo impacto das gotas de chuva sobre o solo, e a segunda é devido ao excesso da energia cisalhante do escoamento sobre a superfície. Além da desagregação, o modelo estima o seu transporte, e quando houver mais sedimento do que a capacidade do escoamento em transportá-lo o modelo passa a estimar a deposição.

\subsection{Equação de Erosão Utilizada pelo Modelo WEPP}

A equação básica para a erosão total é (Foster et al., 1989, citados por Chaves, 1992):

$$
\delta \mathrm{G} / \delta \mathrm{x}=\mathrm{K}_{\mathrm{r}}\left(\mathrm{t}-\mathrm{t}_{\mathrm{c}}\right)\left(1-\mathrm{G} / \mathrm{T}_{\mathrm{c}}\right)+\mathrm{K}_{\mathrm{i}} \mathrm{I}^{2}
$$

em que $\mathrm{G}(\mathrm{kg} / \mathrm{m} . \mathrm{s})=$ carga de sedimento em um ponto $\mathrm{x}$ (m) da encosta; $\mathrm{K}_{\mathrm{r}}(\mathrm{s} / \mathrm{m})$ = parâmetro de erodibilidade em sulcos; $\mathrm{t}(\mathrm{Pa})=$ tensão de cisalhamento que atua nas partículas de solo; $\mathrm{t}_{\mathrm{c}}(\mathrm{Pa})=$ parâmetro de resistência ao cisalhamento; $\mathrm{T}_{\mathrm{c}}(\mathrm{kg} / \mathrm{m} . \mathrm{s})=$ capacidade de transporte de sedimento do escoamento; $\mathrm{K}_{\mathrm{i}}\left(\mathrm{kg} / \mathrm{m}^{4} . \mathrm{s}\right)$ = parâmetro de erodibilidade entre sulcos; e I $(\mathrm{m} / \mathrm{s})=$ intensidade da chuva.

Os parâmetros $\mathrm{K}_{\mathrm{i}}$, $\mathrm{K}_{\mathrm{r}}$ e $\mathrm{t}_{\mathrm{c}}$ devem ser determinados experimentalmente para cada solo, através de experimentos em parcelas de perda de solo com chuva simulada, ou em parcelas com chuva natural; neste caso através de técnicas de otimização.

$\mathrm{O}$ componente $\mathrm{K}_{\mathrm{i}} \mathrm{I}^{2}$ é a contribuição ao sulco das áreas entre sulcos (erosão entre-sulcos). A tensão de cisalhamento t é estimada através da equação $t=P R ~ S$, em que $\mathrm{P}\left(\mathrm{N} / \mathrm{m}^{3}\right)$ é o peso específico do escoamento em uma dada seção; R (m) é o raio hidráulico; e $\mathrm{S}(\mathrm{m} / \mathrm{m})$ é a declividade média da seção. A capacidade de transporte $\mathrm{T}_{\mathrm{c}}$ é estimada através de uma simplificação da equação de Yalin, que utiliza, entre outros fatores, a tensão de cisalhamento (t) sobre o solo.

Quando $\mathrm{G}>\mathrm{T}_{\mathrm{c}}$, ou seja, quando houver condições de deposição no sulco, essa será estimada por:

$$
\delta \mathrm{G} / \delta \mathrm{x}=(\mathrm{B} \mathrm{V} / \mathrm{q})\left(\mathrm{T}_{\mathrm{c}}-\mathrm{G}\right)
$$

em que $\mathrm{B}$ (adimensional $\approx 0,5)=$ coeficiente de turbulência induzida pelos impactos das gotas de chuva; $\mathrm{V}_{\mathrm{f}}(\mathrm{m} / \mathrm{s})=$ velocidade de sedimentação de uma certa classe (diâmetro) de sedimento; e q $\left(\mathrm{m}^{2} / \mathrm{s}\right)$ = vazão por largura unitária do sulco.

Os valores negativos obtidos na equação anterior indicam deposição.

Dessa forma, o modelo estimará os processos hidrossedimentológicos ocorrentes na vertente, prevendo, entre outros, o volume de escoamento superficial, a vazão de pico, a perda de solo e a deposição média na vertente, 
a perda e deposição máximas e suas posições, o aporte de sedimentos (que deixa a vertente) e a relação de enriquecimento de finos do sedimento. Essas previsões podem ser tanto em nível de evento, de média mensal ou de média anual (Chaves, 1992).

As previsões do modelo WEPP oferecem um detalhamento bem maior do que a simples perda de solo média anual, como é o caso da maioria dos modelos de erosão. Esse detalhamento, por sua vez, permite ao planejador ou extensionista a escolha e o dimensionamento mais seguro e preciso de práticas conservacionistas, além de dar indicações sobre os possíveis impactos ambientais devido ao aporte de sedimentos em áreas a jusante da vertente (Chaves, 1992).

Estudos de validação do modelo, com dados de parcelas tipo USLE, indicam que ele representa eficazmente os principais mecanismos de erosão e sedimentação (Chaves, 1996).

A calibração dos parâmetros de erodibilidade do modelo foi iniciada recentemente no Brasil, por pesquisadores do Instituto Agronômico de Campinas, por meio de experimentos em parcelas de erosão em sulcos. Entretanto, cuidados devem ser tomados com relação à calibração dos parâmetros e à determinação dos valores das variáveis do modelo, inclusive em relação à sua distribuição espacial, em função dos erros a eles associados. Em uma análise de incerteza do modelo Wepp, Chaves e Nearing (1991) concluíram que as incertezas inerentes aos parâmetros e às variáveis do modelo se propagam de forma significativa até as predições, com uma propagação média de erro de $400 \%$, afetando principalmente os valores mais baixos de perda de solo. Tal fato se deve, em parte, à alta não-linearidade das equações do modelo, aliada à natureza booleana de sua estrutura.

O objetivo do trabalho foi testar o modelo WEPP (Water Erosion Prediction Project), através de comparações entre volume de enxurrada e perda de solo observados experimentalmente, provenientes dos segmentos de estradas florestais submetidas à chuva natural com inclinações de 1 e $7 \%$ e comprimentos de rampa de 20 e $40 \mathrm{~m}$, e aqueles preditos pelo aplicativo, visando o desenvolvimento de um modelo brasileiro de predição de erosão em estradas florestais.

\section{MATERIAL E MÉTODOS}

O experimento foi realizado no projeto de reflorestamento, povoamento de Pinus caribaea e Pinus oocarpa, com 4 anos de idade, pertencente à tipologia de maciços florestais homogêneos, situado na fazenda Monte Alegre, em Agudos-SP, no período de julho de 2000 a março de 2001. A área de estudo apresentou um relevo predominantemente plano e o solo foi caracterizado como Latossolo Vermelho-Escuro - fase arenosa.

O experimento foi implantado em estradas florestais existentes, sem revestimento primário, compactadas apenas pela ação mecânica do tráfego. $\mathrm{O}$ experimento foi construído segundo as normas da empresa (Figura 1).
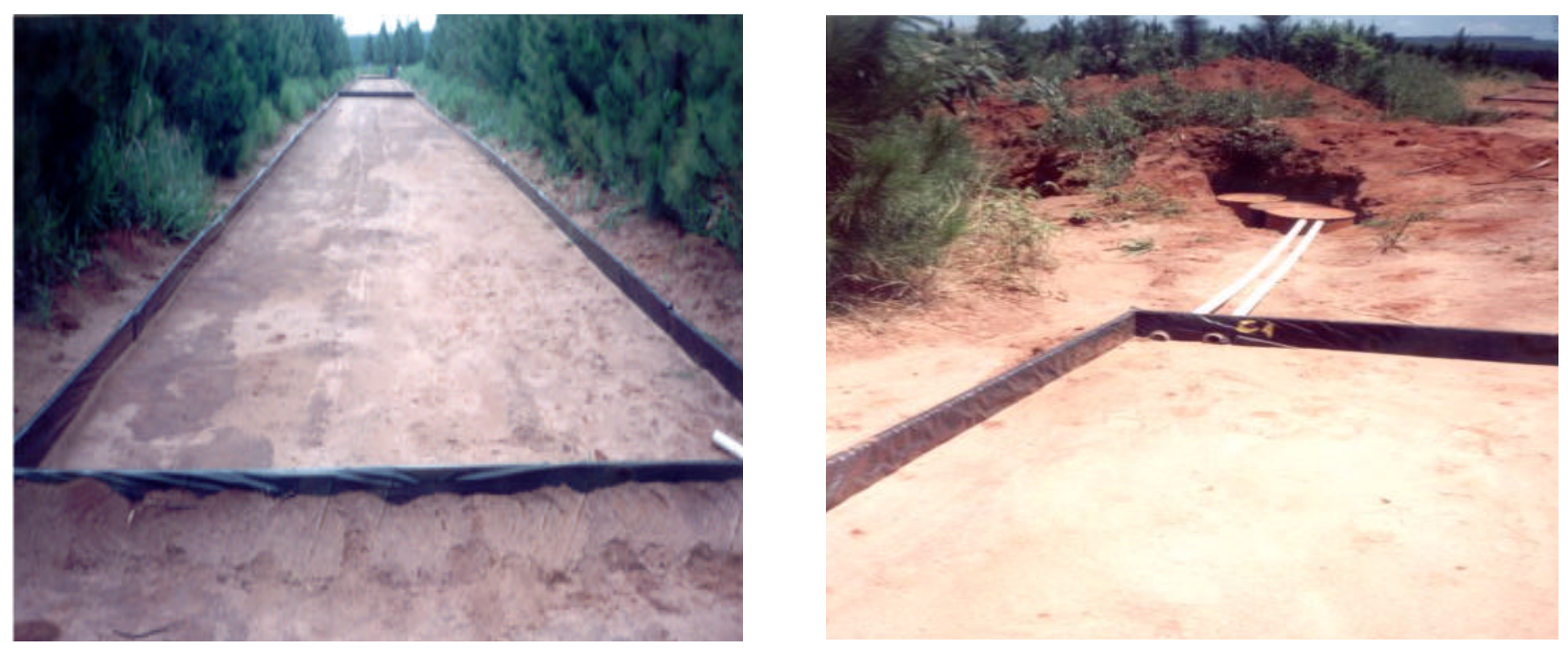

Figura 1 - Segmento de estrada florestal e coletores de água de chuva.

Figure 1 - Forest road segment and rain water collectors. 
As inclinações dos segmentos de estrada de 1 e $7 \%$ foram analisadas, enquanto os comprimentos variaram de $20 \mathrm{e}$ $40 \mathrm{~m}$, com $4 \mathrm{~m}$ de largura. Os segmentos de estrada foram delimitados com tábuas de 0,30 m de largura, envolvidas em lona plástica, visando sua impermeabilização.

Para determinação da quantidade do material erodido foram instalados tambores coletores, com capacidade de 209,25 litros, localizados na parte inferior das estradas, onde foram inseridas tubulações de PVC de 2 polegadas, para coleta dos sedimentos provenientes da estrada propriamente dita. Nos tambores coletores foram feitos orifícios nivelados e perfeitamente iguais, posicionados a $0,65 \mathrm{~m}$ do fundo do primeiro e a $0,60 \mathrm{~m}$ do fundo do segundo, que funcionaram como um divisor Geib. Nas parcelas de 20 e 40 m de comprimento foram feitos cinco e sete orifícios, respectivamente, no primeiro e segundo tambores. $\mathrm{O}$ terceiro tambor foi utilizado para coletar o excedente da enxurrada proveniente do segundo tambor. Os tambores foram ligados em série, através de cano PVC de 2 polegadas.

Os dados de volume e intensidade de precipitação diária foram obtidos com a instalação de pluviômetro e pluviógrafo no local. O período de coleta de dados foi de um ano, concentrando-se na época das chuvas.

Posteriormente, os arquivos de clima, precipitação, solo, inclinação e comprimento do segmento foram introduzidos e adaptados ao modelo de predição de erosão WEPP com o propósito de dar validação ao mesmo, visando a confecção de um modelo apropriado às condições florestais brasileiras.

\section{RESULTADOS E DISCUSSÃO}

\subsection{Comparação entre Valores Observados e Valores Preditos pelo WEPP}

O somatório das precipitações observadas foi utilizado na determinação do volume de enxurrada e peso do solo nas predições do modelo WEPP. Os valores de volume de enxurrada e peso do solo preditos pelo modelo WEPP e os dados observados estão no Quadro 1.

Nas Figuras 2 e 3, podem ser observadas as comparações do volume de enxurrada e peso do solo entre os dados observados e aqueles preditos pelo modelo WEPP nos diferentes tratamentos: C20D1 (comprimento de 20m e declividade de 1\%), C20D7 (comprimento de $20 \mathrm{~m} \mathrm{e}$ declividade de 7\%), C40D1 (comprimento de $40 \mathrm{~m} \mathrm{e}$ declividade de 1\%) e C40D7 (comprimento de $40 \mathrm{~m} \mathrm{e}$ declividade de $7 \%$ ).
Quadro 1 - Volume de enxurrada (VE) e peso do solo (PS) observados e preditos pelo modelo WEPP nos comprimentos do segmento de 20 e $40 \mathrm{~m}$ e declividade de 1 e $7 \%$

Table 1 - Runoff volume (VE) and weight of soil (PS) observed and predicted by the WEPP model on the segment with length of 20 and $40 \mathrm{~m}$ and slope of 1 and $7 \%$

\begin{tabular}{|c|c|c|c|c|}
\hline Tratamento & $\begin{array}{c}\text { Veobs } \\
\text { (litros) }\end{array}$ & $\begin{array}{c}\text { Vewepp } \\
\text { (litros) }\end{array}$ & \multicolumn{1}{c|}{$\begin{array}{c}\text { Psobs } \\
(\mathrm{kg})\end{array}$} & \multicolumn{1}{c|}{$\begin{array}{c}\text { Pswepp } \\
(\mathrm{kg})\end{array}$} \\
\hline C20D1 & $8.052,86$ & $17.780,10$ & 6,615 & 0,540 \\
C20D7 & $9.114,33$ & $21.871,27$ & 16,628 & 17,747 \\
C40D1 & $12.453,02$ & $35.051,46$ & 11,519 & 1,397 \\
C40D7 & $13.467,72$ & $43.644,78$ & 31,534 & 69,453 \\
\hline
\end{tabular}

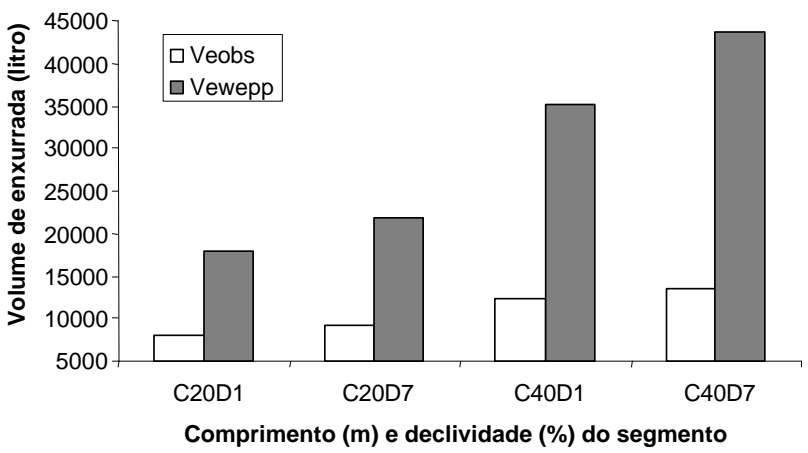

Figura 2 - Volume de enxurrada observado e predito pelo modelo WEPP nos comprimentos do segmento de 20 e $40 \mathrm{~m}$ e declividade de 1 e $7 \%$.

Figure 2 - Runoff volume observed and predicted by the WEPP model on the segment with length of 20 and $40 \mathrm{~m}$ and slope of 1 and $7 \%$.

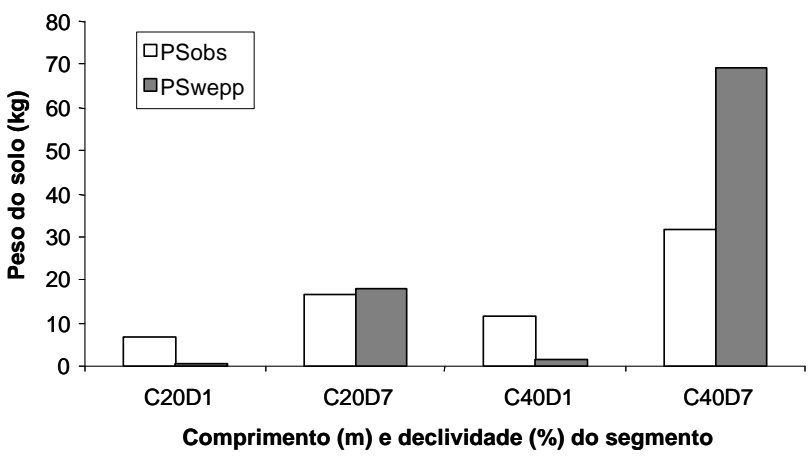

Figura 3 - Peso do solo observado e predito pelo modelo WEPP nos comprimentos do segmento de 20 e $40 \mathrm{~m}$ e declividade de 1 e $7 \%$

Figure 3 - Weight of soil observed and predicted by theWEPP model on the segment with length of 20 and $40 \mathrm{~m}$ and slope of 1 and $7 \%$.

R. Árvore, Viçosa-MG, v.27, n. 3, p.295-300, 2003 
Os valores de volume de enxurrada preditos pelo modelo WEPP nos segmentos de $20 \mathrm{~m}$ de comprimento e $1 \%$ de declividade, $20 \mathrm{~m}$ de comprimento e $7 \%$ de declividade, $40 \mathrm{~m}$ de comprimento e $1 \%$ de declividade e $40 \mathrm{~m}$ de comprimento e $7 \%$ de declividade foram, respectivamente, $120,79,139,97,181,47$ e $224,07 \%$ maiores que os observados.

Os valores observados de peso do solo nos segmentos de $20 \mathrm{~m}$ de comprimento e $1 \%$ de declividade e $40 \mathrm{~m}$ de comprimento e $1 \%$ de declividade foram, respectivamente, 1125,09 e $724,41 \%$ maiores que aqueles fornecidos pelo modelo WEPP. No entanto, os valores de peso do solo preditos pelo modelo WEPP nos segmentos de $20 \mathrm{~m}$ de comprimento e $7 \%$ de declividade e $40 \mathrm{~m}$ de comprimento e $7 \%$ de declividade foram 6,73 e 120,25\% maiores que os observados. Essas diferenças demonstram a necessidade de calibração das variáveis do modelo, uma vez que os arquivos de solo e clima utilizados foram adaptados para a área experimental. Observa-se, no Quadro 1, que o modelo comportou-se de forma não-satisfatória e com predições de perda de solo extremamente adversas das observadas na menor declividade; possivelmente em terrenos planos ele se torna ineficiente, subestimando as perdas.

Os dados de perda de solo preditos pelo modelo foram, em sua maioria, discrepantes dos observados, aproximando-se significativamente apenas no comprimento do segmento de $20 \mathrm{~m}$ e declividade de $7 \%$. No entanto, o modelo comportou-se de forma aceitável na predição de volume de enxurrada, apesar da diferença média de 166,58\% em relação aos dados observados.

\section{CONCLUSÕES}

Os resultados das análises permitiram fazer as seguintes conclusões:

- Na validação do modelo WEPP, os valores preditos de volume de enxurrada foram em média $166,58 \%$ superiores aos dados observados.

- Os valores observados de peso do solo na declividade de $1 \%$ apresentaram variação superior de $1125,09 \%$ no segmento de estrada com $20 \mathrm{~m}$ de comprimento e
$724,41 \%$ no segmento com $40 \mathrm{~m}$ de comprimento, em relação aos valores preditos pelo modelo WEPP, devendo ser ressaltado que nas menores declividades o modelo comportou-se de forma não-satisfatória, subestimando as perdas.

- Os valores de peso do solo preditos pelo modelo WEPP na declividade de $7 \%$ apresentaram variação superior de $6,73 \%$ no segmento de estrada com $20 \mathrm{~m}$ de comprimento e $120,25 \%$ no segmento com $40 \mathrm{~m}$ de comprimento, em relação aos valores observados.

- O modelo brasileiro de predição de erosão pela água denominado WEPP - Brasil não se encontra devidamente calibrado para as nossas condições.

\section{REFERÊNCIAS BIBLIOGRÁFICAS}

ANGULO FILHO, R. et al. Utilização do modelo "WEPP" para simulação de erosão no Brasil - Estudo preliminar. Piracicaba: ESALQ, 1992. p. 65-79. (Relatório Técnico, 3)

CHAVES, H. M. L. O modelo WEPP e sua aplicação no Brasil: I. Descrição do modelo. Campinas: IAC, 1992. p. 41-43 (Boletim Informativo).

CHAVES, H. M. L. Modelagem matemática da erosão hídrica: passado, presente e futuro. In: ALVAREZ V. H.; FONTES, L. E.; FONTES, M. P. F. (Eds.). O solo nos grandes domínios morfoclimáticos do Brasil e o desenvolvimento sustentado. Viçosa: SBCS, UFV, DPS, 1996. p. $731-750$.

CHAVES, H. M. L.; NEARING, M. A. Uncertain analysis of the WEPP soil erosion model. Transitions of Americam Society of Aagriculture Engineering, v. 34, n. 6, p. 2437-2444, 1991.

ELLIOT, W. J.; FOLTZ, R. B.; LUCE, C. H. Predicting the impacts of forest roads on the environment. In: SIMPÓSIO BRASILEIRO SOBRE COLHEITA E TRANSPORTE FLORESTAL, 3., 1997. Anais... Vitória: 1997. p. 99-119.

FERNANDES, E. N. Sistema inteligente de apoio ao processo de avaliação de impactos ambientais de atividades agropecuárias. 1997. 122 f. Tese (Doutorado em Ciência Florestal) - Universidade Federal de Viçosa, Viçosa, 1997. 